\title{
On the education of mathematics teachers
}

\author{
H. Wu
}

Department of Mathematics \#3840

University of California, Berkeley

Berkeley, CA 94720-3840

USA

http://www.math.berkeley.edu/ wu/

wu@math.berkeley.edu

"The teacher preparation curriculum is weighted heavily with courses in 'education methods' at the expense of courses in subject to be taught."

- A Nation at Risk ([NAR])

"Half of the newly appointed mathematics, science, and English teachers are not qualified to teach these subjects..."

- A Nation at Risk ([NAR])

$\S 1$. The importance of content knowledge

$\S 2$. Re-examination of standard upper division courses

$\S 3$. Some proposed changes

$\S 4$. An experiment

$\S 5$. On problem-solving

$\S 6$. What teachers in K-5 need

$\S 7$. Relation with the mathematics education reform

\footnotetext{
${ }^{0}$ March 10, 1997

I am indebted to Richard Askey, Deborah Loewenberg Ball, Paul Clopton, Ole Hald, Ken Ross, and Andre Toom for valuable comments.
} 


\section{The importance of content knowledge}

It would be non-controversial to state that a successful math education reform must comprise improvements in at least three areas: (1) the mathematical qualification of the teachers, (2) the mathematical coherence of the curricula, and (3) the attitude towards learning on the part of students. In this article, I will address only the first problem: how to provide better mathematical education for the teachers. In the process, I will also address the larger issue of how to better educate our math majors in general.

The reader may well ask: does this imply I consider the teachers' pedagogical equipment irrelevant to the quality of instruction in school mathematics? Not at all. But to the extent that pedagogy has been over-emphasized in schools of education whereas content knowledge ${ }^{1}$ is all but ignored (see the first quote from A Nation at Risk above and the book by Rita Kramer $([\mathrm{K}])$ ) the intent of this article is to restore a semblance of balance to the teaching of mathematics.

It is obvious, but it needs to be said, that any discussion of teachers' qualification without a concomitant discussion of their low salaries and their lack of time and incentive for professional development is inherently incomplete. Nevertheless, within the space I allow myself, this article must be confined to the discussion of the qualification issue alone.

A future math teacher may join the profession with only a very meager knowledge of mathematics even under the best of circumstances. For example, one could be credentialed as a math teacher in California even if one's knowledge of calculus consists of no more than one year of freshman calculus (the usual formula-laden variety, not mathematical analysis). When teachers with such a mathematical background are assigned to teach AP calculus to high school students, the results are predictable. This problem cannot be peculiar to California alone and is most likely nation-wide. There is thus a pressing need to tighten up the standards for the credentialing of teachers. I

\footnotetext{
${ }^{1}$ There seems to be some confusion in the use of the term content knowledge at the moment. Some educators tend to think of it as "an inventory of formulas and facts", so that this term tends to connote memorization without understanding. On the other hand, when mathematicians say students should know a certain fact, they have in mind not only that the latter should know the precise statement, but also the reason why it is true (formal proof as well as intuitive idea) and how to use it. Thus content knowledge as used in this article includes what the educators call "facts, conceptual understanding, and problem solving ability". See also the discussion in $\S 5$.
} 
must confess that I am not equipped to tackle a problem of this magnitude. What I propose to do instead is to discuss how we can better educate our future high school and junior high math teachers by improving the teaching of upper division math courses.

Let me begin by arguing briefly why a lesser mathematical preparation for math teachers simply won't do. There are ample reasons to believe that at present most teachers are operating at the outer edge of their mathematical knowledge. Now when one finds oneself in that situation, one is prone to being tense and inflexible, and is consequently not likely to create a friendly atmosphere for learning. There should be a study to look into how much of the so-called math phobia in this country can be traced to this fact (especially in elementary schools). The other simple reason is that no matter how elementary the topic, some students would bring up deep or at least non-elementary related questions. ${ }^{2}$ If the teacher fails to answer such questions too often, the students' confidence in the teacher is eroded and, again, a non-productive learning atmosphere would result.

Along this line, I wish to state my personal conviction that the present depressing state of math education resulted as much from mathematically unqualified teachers as from unqualified curricula. This is hinted at in $A$ Nation At Risk ([NAR]), cf. the second quote at the beginning of this article. In any case, I have never ceased to observe proofs of this fact almost daily. In a posting (1996) in the semi-public e-net mathed, Andre Toom railed against the infamous call for "decreased attention" on "word problems by type" on p.127 of the NCTM Standards ([NC]). His point was that there was nothing wrong with those problems per se, and that they are singled out as scapegoats for failed mathematics education only because too many unqualified teachers do not know enough to handle them. To my mind, he was absolutely correct. Another example of how mathematical inadequacy can ruin mathematics education is given in Alan Schoenfeld's interesting article ([S]). It describes how a teacher, who is a practioner of all the prescribed good pedagogical practices, completely trashed his teaching of Euclidean geometry by his rigid insistence on how two-column proofs must be written up, and on irrelevant issues having to do with pictorial accuracy in doing geometric construction problems. The broad conclusion drawn by Schoenfeld was that "research

\footnotetext{
${ }^{2}$ I have had such experiences firsthand. Once I tried to explain the basics of a parabola to a young boy, but soon found myself explaining loaded cables and freely hanging cables, and the relative growth rates of the parabola and the catenary when he asked if the cable of the Golden Gate Bridge was a parabola.
} 
on the psychology of teaching and learning needs to be expanded both in scope and in breadth....explorations of learning also need to become more focused and detailed as we begin to elaborate on what it means to think mathematically". I would like to add more precision to this conclusion. It seems obvious to me that the main problem which led to such strange instructional manifestations is the excessive amount of emphasis placed on pedagogy per se without regard to mathematical substance in both the way teachers are trained and the way their performance is evaluated. There is also the larger issue of institutional neglect of content knowledge in the educational establishment which led to the empty emphasis on pedagogy. (I refer the reader again to the first quote at the beginning of this article from A Nation At Risk and to ([K]).) The teacher was perhaps doing no more than following the call of this misplaced emphasis. Therefore, a more pertinent conclusion to draw from this case study might be that mathematical content knowledge should not be divorced from pedagogy, and that the training of teachers must place equal emphasis on both.

\section{Re-examination of standard upper division courses}

Because the most substantive portion of a college student's mathematics education takes place in upper division mathematics courses, we must take a close look at the way we have been teaching these courses.

Our undergraduate education is built on the principle of delayed gratification. We mathematicians look at upper division courses as the first steps of a journey of ten thousand miles: in order to prepare students for future research, we feed them technicality after technicality because they need a firm foundation to build on. If they don't understand some of the things we teach them right now, well, they will when they get to graduate school or, if necessary, a few years after they start their research. Then they will put everything together. On the other hand, a conservative estimate is that, nation-wide, at most $20 \%$ of the math majors continue with graduate work in mathematics. The remaining $80 \%$ - the overwhelming majority - look at the last two years of college as the grand finale of their mathematical experience. They expect to sample the best we have to offer. There is thus a classic mismatch of expectations. Take for instance the standard one semester course on complex functions. At the end of such a course, it is perfectly feasible to explain the meaning and significance of the Riemann Mapping theorem, the Dirch- 
let problem, and the Riemann Hypothesis. Imbedded in these three topics are ideas that have changed the course of mathematics in the past century and a half, and are therefore ideas to which those who are about to leave mathematics - the math majors in question - should be exposed, if only in a limited way. Yet in the normal course of events, these ideas are hardly ever mentioned, even if on rare occasions the Riemann Mapping Theorem is stated and proved. Instead, much time is devoted to proving the general form of the Cauchy theorem and other technical facts, for example. At the end, math majors come out of the edifice we call mathematics year after year, having been shown only the nuts and bolts but never a glimpse of its splendor or even the raison d'être of some of its interior designs. We have let them down. What is wrong with this scenario is not that we try to nurture future mathematicians, but that we have neglected the education of the majority who put themselves under our care. They too are part of our charge.

The usual defense of this philosophy of education is that if we give the students a firm technical grounding by presenting them with abstract theorems and rigorous proofs, then they gain the necessary perspective and overall understanding of the details by reading on their own. At some point they will also look back on the elementary mathematics they learned imperfectly in school and pull all the pieces together. Enlightenment ensues. Or so the theory goes.

This is what I call the Intellectual Trickle-down Theory of Learning: aim the teaching at the best students, and somehow the rest will take care of themselves. In practice, however, most of the students who do not go on to graduate school in mathematics are not among those with a strong enough interest or firm enough mastery of the fundamentals to dig deeper for further understanding. Consequently, the college education of these students is long on technical details that they cannot digest but short on the minimal essential information that would enable them to survey the elementary facts from "an advanced standpoint". They go out into the world impoverished in both technique and information for the simple reason that we never had them in mind when we designed our curriculum. Does this perhaps partially explain why our school teachers are not technically sound? ${ }^{3}$

Let me illustrate the preceding discussion with a concrete example. In

\footnotetext{
${ }^{3}$ I emphasize that I am talking about the generic case, and am not trying to stigmatize all students, least of all the present or future school teachers, who do not go on to graduate work. I am in contact with too many excellent school teachers to do that.
} 
the spring semester of 1996, I taught a course in introductory algebra which was attended by math majors who did not intend to continue with graduate study in mathematics. Some were prospective school teachers. Before I discussed the field of rational numbers, I asked how many of them knew why $1 /(1 / 5)=5$. I waited a long time, but not a single hand was raised while a few shook their heads. ${ }^{4}$ One cannot understand the significance of this fact unless one realizes that the subject of fractions is one of the sore spots in school mathematics education. In the article [C], Herb Clemens related the following story:

Last August, I began a week of fractions classes at a workshop for elementary teachers with a graph paper explanation of why $\frac{2 / 7}{1 / 9}=$ $2 \frac{4}{7}$. The reaction of my audience astounded me. Several of the teachers present were simply terrified. None of my protestations about this being a preview, none of my "Don't worry" statements had any effect.

Or, take another statement from p.96 of the NCTM Standards ([NC]):

This is not to suggest, however, that valuable instruction time should be devoted to exercises like $\frac{17}{24}+\frac{5}{18}$ or $5 \frac{3}{4} \times 4 \frac{1}{4}$, which are much harder to visualize and unlikely to occur in real-life situations.

This suggestion concerning the teaching of fractions occurs in the Standard on Estimation and Computation in Grades 5-8 of [NC]. It is difficult for a mathematician to imagine that students going into high school (9th grade) would have trouble computing simple products and sums such as above, but this difficulty evaporates as soon as we look into how fractions are taught in grades 4-8. Take the standard Addison-Wesley Mathematics for Grades 4-8, for example $([\mathrm{EI}])$. There the definition of the addition of fractions unnecessarily brings in the LCM of denominators, and the division of fractions is defined using circular reasoning (for the latter, see p.232 of Grade 6, p.182 of Grade 7 and p.210 of Grade 8), to name just two problems off-hand. The topic of math phobia has been mentioned above, but now I will be more specific and conjecture that in fact fractions are responsible for much of this phobia. Unfortunately, students do not get to learn substantially more about

\footnotetext{
${ }^{4}$ My colleague Ole Hald has suggested to me that one explanation of the lack of response could be the lack of a proper context for the students to understand such a question.
} 
the rational number system in high school because, once there, they take algebra which assumes they know how to compute with rational numbers. Thus by the time students come to the university, very often their understanding of the rationals remains in the primitive state reflected in the two quotations above. It was for this reason that I posed the question concerning $1 /(1 / 5)$ because I wanted to have my suspicions settled once and for all.

In view of this glaring weakness in our average math major's mathematical arsenal, let us take a look at what he or she is taught about the rationals in a typical course in introductory algebra. We first introduce the notion of an integral domain $D$, and construct out of $D$ its quotient field by introducing equivalence classes of ordered pairs $\{(p, q)\}$, show that addition and multiplication among these equivalence classes are well defined and that they form a field $F$. Then we define the canonical injective homomorphism from $D$ to $F$, identify $D$ with its image, and explain to the students that henceforth all nonzero elements of $D$ will have multiplicative inverses in $F$. After all that, we will mention that if we replace $D$ by the integers, then the $F$ above would be the field of rational numbers. Whether or not one would explicitly point out the relationship between the common assertion $1 /(1 / b)=b$ and the general fact that $\left(b^{-1}\right)^{-1}=b$ would depend very much on the instructor, and in any case, even if this is done, it would be a passing remark and no more. In the meantime, the average math major is overwhelmed by this onslaught of newly acquired concepts: integral domain, field, equivalence class, injection, and homomorphism. To most beginners, these are things at best half understood. Thus expecting them to come to grips with the rational numbers by way of the concept of a quotient field is clearly no more than a forlorn hope. It should therefore come as no surprise that, in the midst of such uneasiness and uncertainty, the average student would fail to gain any new insight into such fundamentals as $1 /(1 / b)=b$ or $(-p)(-q)=p q$.

The reason we went into the preceding discussion of what the average student would likely miss in a normal upper divisional mathematics course is the observation made in A Nation At Risk ([NAR]), that "Too many teachers are being drawn from the bottom quarter of graduating high school and college students". The preceding discussion together with this observation would seem to go a long way towards explaining why so many of our teachers come out of four years of college without knowing more about the rationals than what they learned (badly) in school mathematics. And, of course, neither will their students. 


\section{Some proposed changes}

One way to resolve the difficulty concerning the education of our prospective teachers is obviously to teach them (and other math majors who do not go on to graduate work in mathematics) in a separate track. Such a proposal has been made many times in the past, informally if not formally. The main obstacle is that most institutions do not have the resources to teach two separate tracks for each course. This is the bottom line. Beyond this, there is always the suspicion that it is really infra dig for a "good" department to offer "watered down" courses to its own majors. Thus even if money is available, fighting this prejudice would be a formidable task. However, the technical inadequacy of our math teachers is too serious a problem to be glossed over by institutional or professional prejudices. It is time that we meet this problem head on by discussing it in public.

There is perhaps no better explanation of why "different" is not the same as "watered down" than to list what I believe to be the desirable characteristics of courses designed for students who do not pursue graduate work in mathematics.

(1) Only proofs of truly basic theorems are given, but whatever proofs are given should be complete and rigorous. On the one hand, we are doing battle with time: given that there are many topics we want the students to be informed about, the proofs of some of our favorite theorems (e.g. the Jordan canonical form in linear algebra) would have to go in favor of other issues of compelling interest (e.g. historical background or motivation). On the other hand, we also want them to understand that proofs are the underpinning of the edifice we call mathematics. Thus any time we present a proof, we must make sure that it counts.

(2) In contrast with the normal courses which are relentlessly "forwardlooking" (i.e., the far-better-things-to-come in graduate courses), considerable time should be devoted to "looking back". In other words, there should be an emphasis on shedding light on elementary mathematics from an advanced viewpoint. One example is the cleaning up of the confusion about rational numbers (cf. the discussion in the preceding section). Another is the elucidation of Euclidean geometry and axiomatic systems.

(3) Keep the course on as concrete a level as possible, and introduce abstractions only when absolutely necessary. The potency of abstract considerations should not be minimized, but we have to be aware of the point of minimal return, when any more abstraction would decrease rather than pro- 
mote students' understanding. The example of the construction of a quotient field from an integral domain in the context of rational numbers has already been given above.

(4) Ample historical background should be provided. This idea is by now so widely accepted that no argument need be given. One can find no better illustration of this need than the introduction of Dedekind cuts in the definition of real numbers. Among students who are not budding mathematicians, I do not ever recall hearing that they understand this concept. Would they not understand it better if we quote from Dedekind's original essay which contains an explicit statement of his indebtedness to Eudoxus for the idea, and to explain to them the Greeks' crisis concerning rational numbers which then motivated the approach adopted by Eudoxus? One can go on to show them the original passage from Euclid's Book V which is based on Eudoxus' work, and let them see how close it came to the modern definition of a Dedekind cut. This may make Dedekind cuts more palatable to at least the average math majors.

(5) Provide students with some perspective on each subject, including the presentation of surveys of advanced topics. For example, in discussing diagonalization of matrices, students would benefit from a discussion of the various canonical forms - without proofs - because they need to understand that diagonalization is not an isolated trick but a small part of the general attempt to simplify and classify all mathematical objects. Along this line, I cannot help but be amazed by the general tendency in most texts to refrain from discussing any topic that is "out of logical order". The desire to develop the subject $a b$ initio is well taken, but what is there to fear about exposing students to interesting advanced ideas without proofs, so long as the advanced nature of the material is made clear to them? The way we learn is hardly "logically-linear"; otherwise there would be no incentive for any of us to go to colloquium lectures. So why deny the students the same opportunity to learn when we can so easily provide it?

(6) Give motivation at every opportunity. The usual complaint about school mathematics degenerating into rote-learning is fundamentally a reflection of the fact that most teachers were themselves never exposed to any motivation for every concept, lemma, and theorem that they learned. It is incumbent on the college instructors to break this vicious circle. 


\section{An experiment}

What I will take up next is the description of a small experiment I am currently conducting at Berkeley in an attempt to implement the preceding philosophy of teaching. Starting in the spring semester of 1996, I have been offering an upper division course each semester specifically for "math majors who do not go on to graduate school in mathematics". At the time of writing (February 1997), I have taught introductory algebra and linear algebra, and am currently teaching differential geometry. The remaining courses I hope to have taught by June of 1999 (in some order) are history of mathematics, introductory analysis, complex functions and classical geometries. This choice of courses is dictated partly by my own limitations and partly by the fact that they are among the courses most likely to be taken by future school math teachers. Having taught two such courses and having planned a third with some care, I would like to recount my experience in some detail. Since there is as yet no tradition of teaching upper divisional math courses with this philosophical orientation, others might find this account to be of some value.

Two general observations emerged from this experience. The first one is that it is very difficult, if not impossible, to find an appropriate text for such a course. Almost all the standard texts are written to prepare students for graduate work in mathematics. At the other extreme are a few texts that try to be "user-friendly" by trivializing the content. Neither would serve my purpose. The other observation is that in this approach to upper divisional instruction, the trade-offs are quite pronounced even for someone who is prepared for them. Let me be more specific by discussing separately the two courses I have taught thus far.

For the introductory algebra course, I announced that the goals of the course were the solutions of the three classical construction problems and the explanation of why the roots of certain polynomials of degree $\geq 5$ cannot be extracted from the coefficients by use of radicals. In more details, the first two thirds of the course were devoted to the following topics:

Part I: The quadratic closure of $\mathbb{Q}$, constructible numbers, $\mathbb{Z}$ and $\mathbb{Q}$ revisited, Euclidean algorithm and prime factorization, congruences $\bmod n$, fields, polynomials over a field, irriducibility and unique factorization of polynomials, Eisenstein, complex numbers and the fundamental theorem of algebra, roots of unity and cyclo- 
tomic polynomials, field extensions and their degrees, solutions of the construction problems, constructibility of regular polygons.

The last third of the course was more descriptive in nature. It consisted of the following:

Part 2: Isomorphism of fields, automorphisms relative to a ground field, root fields, computations of automorphisms, groups and basic properties, solvable groups, Galois group of an extension, radical extensions, theorems of Abel and Galois.

The main reason for the decision to direct the whole course towards these classical problems was the hope that students' prior familiarity with these problems would entice them to learn the abstract algebraic concepts needed for their solutions. My hope was not in vain: a few of my 19 students told me after the course was over that they worked harder for this course than other math courses because they cared about what they were learning. The thought occurred to me that with the gutting of Euclidean geometry in the present mathematics education reform and the de-emphasis of purely mathematical questions (without real-world relevance), the day may come when students can no longer be motivated by these famous problems because they will never be exposed to them. This is a potential educational crisis that mathematicians must do all they can to avert.

Another reason for this choice of materials was that, since these problems gave impetus to almost all the important advances in algebra up to the nineteenth century, the course would provide a natural setting to delve into the historical roots of the subject. I got to discuss the long and difficult road to symbolic algebraic notations through the centuries, the work of the colorful pioneers such as Cardan and Tartaglia, the construction of the regular 17-gon and some of Gauss' contributions, and Galois and Abel and their roles in the theory of polynomials and abstract algebra.

Compared with more standard approaches to introductory algebra, some losses and gains are worth noting. Because I consider the topics in Part 1 to be basic to a teacher's understanding of algebra, every theorem there was proved, with the exception (of course) of the fundamental theorem of algebra, the transcendence of $\pi$, and the theorem of Gauss on the constructibility of regular polygons. The students received, and welcomed, a careful and rigorous treatment of $\mathbb{Q}$ and $\mathbb{C}$. In addition, because Part I gives such a detailed treatment of the polynomial ring over $\mathbb{R}$, the students got to understand this 
important object - important for their later needs in life - much better than otherwise possible. For example, I made a point of proving for them the technique of partial fractions which is usually imperfectly stated and used for integration in calculus. On the other hand, this choice of topics entails certain glaring omissions: no PID's and no UFD's, no general construction of the quotient field of a domain, and no serious discussion of ideals, ring homomorphisms and quotient rings.

The omissions in Part 2 are much more serious. With only five weeks to treat these sophisticated topics, there was hardly time to prove any theorem other than the simplest. Even at the most basic level, there was no discussion of homomorphism between groups, and hence also no discussion of the relationship between normal subgroups and the kernel of a homomorphism, and theorems about the existence of subgroups of an appropriate order were hardly mentioned. In exchange, students were given exposure to the fantastic ideas of Galois theory - without proofs, to be sure - and the hope is that perhaps one day some of them would revisit the whole terrain on their own.

Implicit in the preceding syllabus is the fact that no noncommutative mathematics appears until two thirds of the way into the course when groups enter the discussion. This is by design. It seems to me that students taking such a course are confronted with proofs in a serious way for the first time, and that is enough of a hurdle without their being simultaneously overloaded by noncommutativity as well. We should not overlook the fact that it took mankind more than two thousand years after Euclid to face up to noncommutativity, so it seems unfair that beginning students are not given a few weeks of reprieve before being saddled with it. The presentation in almost all the standard texts in algebra begin with groups, to be followed by rings and fields. This is in fact the order adopted by van der Waerden in his pioneering text of 1931 ([WA]). However, van der Waerden was writing a research monograph, and there seems to be very little reason why undergraduate texts should follow exactly the same order without due regard for pedagogy. Along this line, let it be said that the first American undergraduate textbook on (modern) algebra, A Survey of Modern Algebra of Birkhoff and MacLane $([\mathrm{BM}])$, does begin with integers, commutative rings, and more than a hundred pages of commutative mathematics before launching into groups. There is a reason why Birkhoff-MacLane is still in print after 55 years. Having said that, I want to reiterate a serious concern in the teaching of such a course, which is that of getting an appropriate textbook. This is not the place for an extended discussion of this issue except to point out this obvious and 
pressing need.

Next, let us turn to linear algebra. Among upper division math courses, this course may be the only one which is as rich in nontrivial scientific applications as calculus. Furthermore, this is also a subject almost tailor-made for computers and therefore the consideration of computational simplicity plays an important role. For the benefit of those students who do not go on to higher mathematics, a course on linear algebra that emphasizes both of these facts in place of topics like the Cayley-Hamilton theorem and the rational canonical form would seem to be more educational. ${ }^{5}$ Standard courses do pay some attention to the second fact to be sure: they usually begin with the method of Gaussian elimination for the solution of linear systems and the comment is then duly made that this method is superior to the use of determinants even for $n \times n$ systems because far fewer operations are used. However, it is also true that such courses then proceed to drop this consideration of computational simplicity the rest of the way. I would prefer that this consideration be the underlying theme of the whole course because, in scientific applications, savings in cost and time are important. ${ }^{6}$ The syllabus for the course I gave in the fall of 1996 is then:

Elementary row operations, Gaussian elimination, existence and uniqueness of LU decomposition of nonsingular square matrices, approximate solutions of ODE's by linear systems, review of vector spaces and associated concepts, LU decomposition in general, the row space, column space and solution space of a general matrix, the rank-nullity theorem, application to electrical networks, inner product spaces, orthogonal projection on a subspace, least square approximations, Gram-Schmidt, the QR decomposition of a nonsingular matrix, signal processing and the fast Fourier transform, determinants, eigenvalues and eigenvectors, diagonalization, applications to Fibonacci sequence, symmetric and Hermitian matrices, quadratic forms.

This is clearly a course on matrices rather than abstract vector spaces. Even so, some standard topics on matrices are conspicuous by their absence:

\footnotetext{
${ }^{5}$ Of course students should learn the Cayley-Hamilton theorem and the rational form too if there is time.

${ }^{6}$ It should be mentioned that the issue of roundoff errors was mentioned in my course but not pursued. It seems to me that this would be better handled in a course on numerical analysis.
} 
the minimal polynomial, Cayley-Hamilton, Jordan canonical form and rational canonical form. But perhaps the most distressing aspect was my inability, due to lack of time, to impress on the students the advantage of knowing the coordinate-free point of view. It goes without saying that there was no mention in the course of invariant subspaces, dual spaces, and induced dual linear transformations. The difficulty with some of these deficiencies could have been alleviated by making reading assignments in an appropriate textbook, one in which the applications are presented with integrity and the abstract point of view is treated with the clarity and precision that befit a mathematics text. However, the scarcity of textbooks suitable for the kind of teaching under discussion continues to call attention to itself. It is hoped that this lacuna in the literature will soon be filled.

What remains to be said are the gains that go with such losses, namely, the interesting applications that throw a completely different light on abstract linear algebra. Personally, I must admit to having been enthralled by the applications of the fast Fourier transform to signal processing, and this sense of enchantment would be shared by the students too if the latter is properly explained. No less interesting is the way the QR decomposition helps save time and cost in formulating precise experimental laws using the least squares method. These rather pronounced trade-offs in such an approach to teaching will undoubtedly continue to invite strong reactions from each of us.

Finally, an ongoing concern of my mathematical colleagues is that by not proving everything in these courses, students run the risk of getting a distorted perception of the fundamental nature of proofs in mathematics. To allay such fears, I have appended the final exams of the preceding two courses. They should speak for themselves.

\section{On problem-solving}

Whatever approach one adopts in the teaching of upper division courses, the mathematical substance of the courses themselves must not be compromised. With a view towards ensuring that a minimum of topics be covered in these experimental courses, I gave lectures all the way through. Students were told about the desirability of forming study groups among themselves and the need to retrace the steps, whenever possible, that led to the discovery

of the theorems at home. After all this, it remains to address the issue of how students were taught to do mathematics, i.e., what was done about the 
problem-solving aspect of mathematics education?

Among educators, there is presently a tendency to isolate problem-solving as a separate component of mathematics education and cultivate it per se. ${ }^{7}$ However, mathematicians take a different stand on this issue: they place problem-solving right in the context of content knowledge. When a new topic is introduced, related homework problems are given and students are expected to solve them by any one of the three standard strategies: imitate the arguments in the lectures, specialize the abstract arguments to concrete situations, or extend a given argument to more general situations. Of course, there may also be more challenging problems which transcend these three basic strategies, but the overriding idea is that students should master new mathematics as they polish their problem-solving skills. Since as a rule time is not available for group work during classes, it is understood that students have to work by themselves or form their own study groups away from class. There is as yet no valid argument that I am aware of that this conception of mathematics education is less than pedagogically sound, except for one intangble factor: what if the average student does not have the drive or the burning desire to execute these basic strategies unaided? This brings up the unanswerable question of how far must a teacher go in order to beg the students to learn. More to the point, is the professor responsible for students' performance even when the students are reluctant to uphold their end of the bargain, which is to do their best? The answers to these questions used to be fairly straightforward, but in 1997, they are much less so. A more pertinent, and more serious issue is what help is available for those students who try hard but still need reasonable feedback on what is wrong with their solutions and how they can improve. While I have devised my own ad hoc response to this challenge ${ }^{8}$, I cannot help but feel that some effort on the part of the professor beyond that of giving lectures may be needed.

My decision in those two courses was to pass out solutions to all the non-routine homework problems at the time they were due. Because I do not believe that students would benefit from the solutions if they have not thought hard about them in the first place, I also instituted the policy of not giving out excessive hints about the problems' solutions ahead of time. The idea behind the solution sets was that students would not only learn

\footnotetext{
${ }^{7}$ In the same way that conceptual understanding is isolated from technical skill and cultivated per se. Cf. [W1].

${ }^{8}$ As many of my colleagues at Berkeley have.
} 
from them the necessary technical skills but also the proper way to write up a proof, the latter being one of the things I emphasized throughout the semester. Unfortunately, I learned later that perhaps a majority of the students had not bothered to read the solution sets!

Starting with the current semester (Spring 1997), when I am teaching differential geometry, I try a different tack: I schedule two extra (voluntary) problem sessions each week but no longer pass out solution sets. I encourage informal discussions among the students during these sessions, but most of the time I try to explain directly the thought process behind the solution of each problem. Time will tell if this turns out to be a more effective way of reaching out to the students.

The question could be raised as to why I do not pass out solution sets and schedule problem sessions at the same time. One can also ask whether there is a more effective scheme that would help the students learn. I can only answer the second question by saying that I do what is obvious but am ignorant of any fancy pedagogical devices. As to the first question, one way to answer it is to ask another question: why don't all of us offer office hours everyday from nine to five? The truth of the matter is that while we all want to help students learn, each of us must decide how much of ourselves we can give to teaching beyond the normal call of duty. I happen to believe that one should not do too much in this direction because teaching - at least on the university level - should be a collaborative process: both teacher and students must meet each other half way. I have already made the suggestion that additional work beyond the standard duty of giving lectures - such as extra problem sessions, giving out solution sets, or some other variants - may be needed for good education. It remains to be seen how it will be received by the mathematical community at large.

\section{What teachers of K-5 need}

All the preceding comments are about the education of teachers in junior and senior high schools, and therefore indirectly about the teaching of mathematics in these schools. Now I want to say a few words about the teaching of mathematics in K-5. In these grades, the teacher teaches everything, including mathematics. Extensive evidence is available to show that this approach to mathematics education very often produces unhappy results. My personal perception that the phobia of the so-called math-phobics is usually 
well formed by the fifth grade can be easily verified ${ }^{9}$ given the capabilities of modern day education research. Thus the mathematics education of teachers in K-5 is a topic worthy of our most serious considerations. If we accept the fact that the mathematical demands on these teachers are in fact every bit as severe (but different) as those in high school, then the only sensible solution to this educational impasse is to either make more stringent demands on K-5 teachers' mathematical content knowledge or to install mathematics specialists in elementary schools to teach mathematics at least from the third grade onward. One can justify this proposal by pointing to what is done in other countries (e.g., Japan and Taiwan), but that is hardly necessary. What induces math phobia is the tendency of some teachers to teach mathematics by fiat - without recourse to reason - as well as their inability to explain why an algorithm, a technique, or a concept deserves to be learned. One acquires such competence not by attending some elementary skills-oriented courses, as is being done for K-5 teachers - although technical skill is a necessary part of a math teacher's equipment — but by acquiring some mathematical culture beyond the level of basic skills. Such a requirement would seem to far outstrip what we presently require of a typical K-5 teacher.

The description of the kind of mathematical equipment needed by a mathematics teacher in K-5 requires some careful thought and will not be taken up here. When one considers the difficulty in safeguarding a reasonable mathematics education even for the secondary school teachers, it may be a little pre-mature to worry about the details of a good mathematics education for teachers in K-5. Two comments can be made, however. Whatever the optimal mathematics education for K-5 teachers may be, it seems safe to say that their mathematics courses would embody the characteristics announced in §3. Furthermore, inasmuch as the technical requirements for K-5 teachers are not as high as those for secondary school teachers, there should be room for the kind of "mathematics course for poets" exemplified by the text of F.P. Greenleaf $[G]$. This text succeeds in informing the readers about the quantitative aspect as well as the spirit of mathematics with a minimum of technical details. I believe our teachers need more exposure to this kind of materials.

\footnotetext{
${ }^{9}$ Or, less likely, refuted.
} 


\section{Relation with the mathematics education reform}

Let me conclude with two general comments. The first one is that the practical adjustments one has to make in order to teach the kind of courses proposed here are far from simple. For one thing, the normal upper division courses designed for the training of future mathematicians are here to stay because, since a basic function of universities is to be the repository of knowledge, we need mathematicians to keep alive the mathematics of the future. There is thus no justification for advocating that, in the name of the needs of the majority - those whose mathematics education terminates with their B.A.'s - we must teach all our upper division courses only for the majority from now on. Such all-or-nothing extremism is in any case unnecessary because the existing framework is actually flexible enough to accommodate some partial solutions. For example, courses that are terminal in the sense that no other undergraduate course depends on them as pre-requisites can be taught in the alternative way advocated here. ${ }^{10}$ However, basic courses such as introductory analysis or algebra are more problematic. This difficulty is less severe in the bigger universities where such courses are typically offered in parallel sections. Devoting at least one of these sections to the teaching of those majors who do not continue with graduate studies in mathematics would be not only feasible but in fact fully justified. This is incidentally the reason I have been able to conduct my experiment at Berkeley. In the smaller institutions, I have no innovative suggestions to make except to ask that the needs of the majority of our students be kept in mind. Undoubtedly, some ingenious local solutions will always exist so long as the will and the awareness are there.

I hope I have been sufficiently emphatic about the fact that the approach to teaching upper division courses being proposed here is an alternative and not a replacement. I have also been quite precise about what I perceive to be the obvious trade-offs in this approach. Even in my experiment at Berkeley, I have been just as careful in describing its purpose and its limitations. Berkeley has a tradition of making advance course announcements for the benefit of the students, and here is part of what I announced about the course on introductory algebra:

This course is an introductory course in algebra designed specifically for math majors who do not plan on attending graduate

\footnotetext{
${ }^{10}$ Differential geometry and history of mathematics are two that readily come to mind.
} 
school and for future junior high and high school teachers...

This course differs from the usual one in its emphasis: it will slight many of the technical proofs that are more useful for advanced study in mathematics than for a well-rounded mathematical education at this level, and will instead spend the time on more interesting topics that would normally be omitted... .

Because the course covers a wider territory than usual, the fact that it proves fewer theorems must not be mistaken to mean that it is easier. It is a trade-off: the effort to prove technical results will now be spent on comprehending the inter-connections among many topics... .

In the academic context, what I have done is no more than what must be done in order to meet the minimum standard of scholarship. Indeed, any advocate of a new proposal, scientific or otherwise, has to be sufficiently competent to know the range of applicability of his proposal as well as its potential pitfalls. He should also be sufficiently responsible to make explicit all these facts at the outset or as soon as they are known. For example, when a drug is put on the market, there have to be explicit statements about its indications (what it is for) and its dosage (how to use it), as well as a clear warning about when not to use it and what the side-effects are. This then brings me to my second comment, which is this: I wish the current mathematics education reform could be conducted in a way that conforms to this minimum standard.

What brings this to mind is the fact that what is being proposed here namely, a different kind of education for the majority (among math majors) - is fundamentally no different from what the reformers are advocating for K-12 mathematics and the teaching of calculus. Having awakened to the fact that the "traditional" education is not only deficient in crucial ways, but is especially deficient for the majority of students who will not be mathematicians, the reformers made proposals for a different curriculum according to this perception (which I agree with in the main), one that favors heavily the average students and slights the intellectual interests of (say) the upper $20 \%$ (cf. [W1], [W2]). Some would disagree, but it can be argued that the reform has brought some welcome changes to the mathematics education of the majority of the students, especially those in the lower $50 \%$ in terms of mathematical ability or interest. However, instead of stating their case carefully and explicitly, - a case that is clearly not without merits, - the reformers 
go about proselytizing these changes as the right way to teach all students. One looks in vain for a clear statement on the limitations of the proposed changes or, for that matter, a detailed exposition of the pros and cons of any reform curriculum by the reformers themselves. We seem to be in an era where a partial truth is equated with the whole truth and an intellectual advocacy is confused with a political campaign.

For the benefit of future generations - and we are here talking about something that affects the future generations - let us hope that the normal candor of intellectual pursuits will be restored in the arena of education.

\section{A Final Exam of Introductory Algebra}

\section{Math 113 FINAL EXAM \\ May 13, 1996 8-11 am Prof. Wu}

1. (5\%) Prove that for an integer $n, 3|n \Longleftrightarrow 3|$ (sum of digits of $n$ ).

2. (5\%) Let $f(x)=x^{n}+a_{n-1} x^{n-1}+\cdots a_{1} x+a_{0}$ be a polynomial with integer coefficients, and let $r$ be a rational number such that $f(r)=0$. Show that $r$ has to be an integer and $r \mid a_{0}$.

3. $(5 \%)$ Find a minimal polynomial of $\sqrt[3]{1+\sqrt{3}}$ over $\mathbb{Q}$. (Be sure to prove that it is minimal.)

4. $(5 \%)$ Let $n$ be a positve integer $\geq 2$ such that $n \mid\left(b^{n-1}-1\right)$ for all integers $b$ which are not a multiple of $n$. What can you say about $n$ ?

5. (5\%) Do the nonzero elements of $\mathbb{Z}_{13}$ form a cyclic group under multiplication? Give reasons.

6. $(10 \%)$ Let $p$ be a prime.

(a) Prove: $p \mid\left(\begin{array}{l}p \\ k\end{array}\right)$ for $k=1, \cdots p-1$, where $\left(\begin{array}{l}p \\ k\end{array}\right) \equiv \frac{p !}{k !(p-k) !}$.

(b) Prove: the mapping $f: \mathbb{Z}_{p} \rightarrow \mathbb{Z}_{p}$ defined by $f(k)=k^{p}$ for all $k \in \mathbb{Z}_{p}$ is a field isomorphism.

7. $(10 \%)$ Is $x^{4}+2 x+3$ irreducible over $\mathbb{R}$ ? Is it irreducible over $\mathbb{Q}$ ? Give reasons.

8. (10\%) Let $F \equiv\{a+i b: a, b \in \mathbb{Q}\}$ and let $K \equiv \mathbb{Q}[x] /\left(x^{2}+1\right) \mathbb{Q}[x]$. Show that $F$ is isomorphic to $K$ as fields by defining a map $\varphi: F \rightarrow K$ and show that $\varphi$ has all the requisite properties.

9. (10\%) If $\beta$ is a root of $x^{3}-x+1$, find some $p(x) \in \mathbb{Q}[x]$ so that $\left(\beta^{2}-2\right) p(\beta)=1$. 
10. $(10 \%)$ Let $\zeta=e^{i 2 \pi / 3}$. Compute $(\mathbb{Q}(\zeta, \sqrt[3]{5}): \mathbb{Q}(\zeta))$.

11. $(25 \%)$ (In (a)-(d) below, each part could be done independently.)

(a) Assume that if $p$ is a prime, then $x^{p-1}+x^{p-2}+\cdots+1$ is irreducible over $\mathbb{Q}$. Compute $(\mathbb{Q}(\cos (2 \pi / 7)+i \sin (2 \pi / 7))$ : $\mathbb{Q})$. (Each step should be clearly explained.)

(b) Suppose the regular 7-gon can be constructed with straightedge and compass. Explain why $\left(\mathbb{Q}(\cos (2 \pi / 7): \mathbb{Q})=2^{k}\right.$ for some $k \in \mathbb{Z}^{+}$.

(c) If $F \equiv \mathbb{Q}(\cos (2 \pi / 7))$, show that $(F(i \sin (2 \pi / 7)): F)=1$ or 2 .

(d) Use (b) and (c) to conclude that if the regular 7-gon can be constructed with straightedge and compass, then $(\mathbb{Q}(\cos (2 \pi / 7)+i \sin (2 \pi / 7)): \mathbb{Q})=2^{m}$ for some $m \in \mathbb{Z}^{+}$.

(e) What can you conclude from (a) and (d)? What is your guess concerning the construction of the regular 11-gon, the regular 13-gon, the regular 23-gon, etc.?

\section{A Final Exam of Linear Algebra}

\section{Math 110 FINAL EXAM \\ Dec 11, 1996 12:30-3:30 pm Prof. Wu}

1. (5\%) Find the determinant of $\left[\begin{array}{llll}2 & 2 & 0 & 4 \\ 3 & 3 & 2 & 2 \\ 0 & 1 & 3 & 2 \\ 2 & 0 & 2 & 1\end{array}\right]$ and show all your steps.

2. (15\%) Let $A=\left[\begin{array}{ccc}1 & -1 & -1 \\ -1 & 1 & -1 \\ -1 & -1 & 1\end{array}\right]$. Find its eigenvalues and the corresponding eigenvectors. Also find a $3 \times 3$ matrix $S$ and a diagonal matrix $D$ so that $S^{-1} A S=D$.

3. (5\%) If $Q$ is an $n \times n$ orthogonal matrix, what is $\operatorname{det} Q$ ? What are the eigenvalues of $Q$ ? Give reasons.

4. $(15 \%)$ Let $F_{k}$ denote the Fourier matrix of dimension $k$. Define for 
each $n$ :

$$
Y_{2 n}=\left[\begin{array}{c}
y_{0} \\
y_{1} \\
y_{2} \\
\vdots \\
y_{2 n-1}
\end{array}\right], \quad Y_{\text {odd }}=\left[\begin{array}{c}
y_{1} \\
y_{3} \\
y_{5} \\
\vdots \\
y_{2 n-1}
\end{array}\right], \quad Y_{\text {even }}=\left[\begin{array}{c}
y_{0} \\
y_{2} \\
y_{4} \\
\vdots \\
y_{2 n-2}
\end{array}\right]
$$

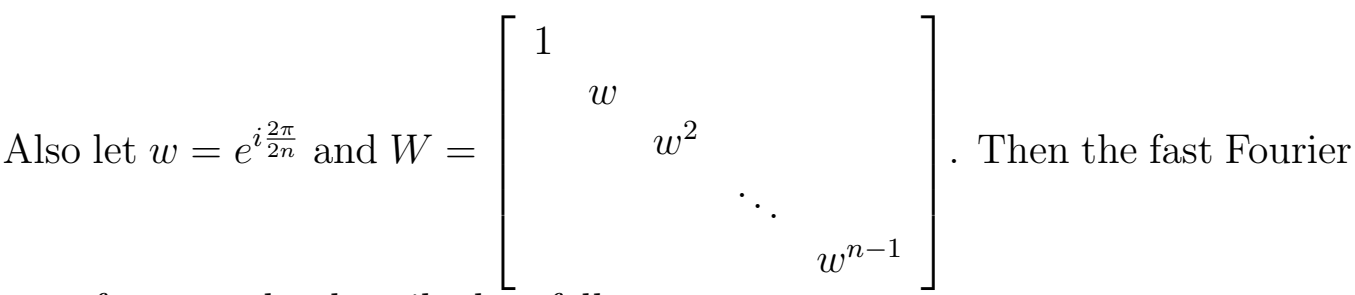

transform can be described as follows:

$$
F_{2 n} Y_{2 n}=\left[\begin{array}{c}
F_{n} Y_{\text {even }}+W F_{n} Y_{\text {odd }} \\
F_{n} Y_{\text {even }}-W F_{n} Y_{\text {odd }}
\end{array}\right]
$$

Now let $\rho(2 n)$ denote the minimum number of operations needed to compute $F_{2 n} Y_{2 n}$. Prove: $\rho\left(2^{k}\right) \leq k 2^{k}$ for every integer $k \geq 1$. (Recall: an "operation" means either a multiplication-and-an-addition or a division.)

5. (10\%) We want a plane $y=C+D t+E z$ in $y-t-z$ space that "best fits" (in the sense of least squares) the following data: $y=3$ when $t=1$ and $z=1 ; y=5$ when $t=2$ and $z=1 ; y=6$ when $t=0$ and $z=3$; and $y=0$ when $t=0$ and $z=0$. Set up, but no not solve the $3 \times 3$ linear system of equations that the least squares solution $C, D, E$ must satisfy.

6. (10\%) Let $v_{1}, \cdots, v_{k}$ be eigenvectors of an $n \times n$ matrix $A$ corresponding to distinct eigenvalues $\lambda_{1}, \cdots, \lambda_{k}$, respectively, where $k \leq n$. Prove that $v_{1}, \cdots, v_{k}$ are linearly independent.

7. (10\%) Suppose a real $n \times n$ matrix $A$ has $n$ distinct real eigenvalues. Is there necessarily a real $n \times n$ matrix $S$ so that $S^{-1} A S$ is diagonal? Explain.

8. (10\%) If the eigenvalues of $A$ are $\lambda_{1}, \cdots, \lambda_{n}$ (not necessarily distinct), what are the eigenvalues of $A^{k}$ where $k$ is an integer $\geq 1$ ? If $A$ is nonsingular, what are the eigenvalues of $A^{-k}$ for $k \geq 1$ ? Give reason.

9. (15\%) Let $P$ be the projection matrix which projects $\mathbb{R}^{n}$ onto a $k$ dimensional subspace $W \subset \mathbb{R}^{n}$, where $0<k<n$. Enumerate all the eigenvalues of $P$ and for each eigenvalue, describe all its eigenvectors.

10. (5\%) Let $A$ be an $n \times n$ matrix and let $A^{\prime}$ be obtained from $A$ be Gaussian elimination. Do $A$ and $A^{\prime}$ necessarily have the same eigenvalues? Give reasons. 


\section{References}

[BM] Garrett Birkhoff and Saunders MacLane, A Survey of Modern Algebra, The Macmillan company, New York, 1941.

[C] C. H. Clemens, Can university math people contribute significantly to precollege mathematics education (beyond giving future teachers a few preservice courses?), CBMS Issues in Mathematics Education, Volume 5, Amer. Math. Soc., 1995, 55-59.

[EI] Robert Eicholz et al., Addison-Wesley Mathematics for Grades 4-8, Addison-Wesley, Menlo Park, CA, 1995.

[G] F. P. Greenleaf, Understanding the Mathematical Patterns of $\mathrm{Na}$ ture, McGraw Hill, New York, 1994.

[K] Rita Kramer, Ed School Follies, The Free Press, 1991.

[NAR] A Nation at Risk, U.S. Department of Education, Washington D.C., 1983.

[NC] Curriculum and Evaluation Standards for School Mathematics, National Council of Teachers of Mathematics, Reston, 1989. Available at the web site:

http://www.enc.org/online/NCTM/280dtoc1.html

[S] Alan Schoenfeld, When good teaching leads to bad results; The disasters of 'well-taught' mathematics courses, Educational Psychologist, 23(1988), 145-166.

[WA] B. L. van der Waerden, Moderne Algebra, J. Springer, Berlin, 193031.

[W1] H. Wu, What about the top 20\%? Manuscript of a talk given at the Annual Meeting of the Amer. Math. Soc., Jan. 4, 1995; available from the author.

[W2] H. Wu, The mathematics education reform: why you should be concerned and what you can do, to appear. 